\title{
Detection of suicide among the elderly in a long term care facility
}

\author{
This article was published in the following Dove Press journal: \\ Clinical Interventions in Aging \\ 18 November 2013 \\ Number of times this article has been viewed
}

\author{
Tinakon Wongpakaran \\ Nahathai Wongpakaran \\ Department of Psychiatry, Faculty \\ of Medicine, Chiang Mai University, \\ Chiang Mai, Thailand
}

Correspondence: Tinakon Wongpakaran Department of Psychiatry, Faculty of Medicine, Chiang Mai University, I 0 Intawaroros Road, Tambon Sriphum, Amphoe Muang, Chiang Mai, 50200, Thailand

Tel +66 53945422

Fax +66 53289219

Email tinakon.w@cmu.ac.th
Purpose: The aim of this study was to establish the level of correlation between the suicide item contained within the Core Symptom Index (CSI), and the presence of suicidal thoughts as assessed by the Mini-International Neuropsychiatric Interview (MINI) and the Cornell Scale of Depression in Dementia (CSDD).

Patients and methods: Seventy elderly residents in a long term care facility were included in this study. All of these patients completed a CSI and a geriatric depression scale (GDS), plus were interviewed using CSDD, MINI (suicide module), and MMSE. Test characteristics of item two of the CSI (suicidal thoughts) and MINI were compared. Gwet's AC1 and Cohen's Kappa were also used to test the level of agreement between raters, and univariate analysis was used to determine predictors for the severity of any suicidal thoughts present.

Results: There was found to be a significant correlation between suicidal ideation, as assessed by item two of the CSI, and the suicidal ideation score as assessed by MINI and CSDD $(r=0.773$ and $r=0.626, P<0.001$, respectively). The level of agreement across all three instruments was good (Gwet's AC1 $=0.907)$. The CSI yielded a high level of sensitivity $(100 \%)$ and specificity $(90.32 \%)$ for suicidal thoughts as measured by MINI, with an area under the curve of $97 \%$. When assessing predictors of the severity of suicidal thoughts, only item two of the CSI predicted severity, while the depression, GDS, and total scores obtained from the CSI did not.

Conclusion: CSI item two has the ability to detect suicidal ideation, regardless of whether the patient has cognitive impairment and/or depression or not, and is currently the best predictor of its presence. Therefore, it shows promise as a measure for screening the presence of suicidal thoughts among the elderly in long term care facilities.

Keywords: core symptom index, elderly, suicidality, suicidal thoughts, long-term care, depression

\section{Introduction}

Suicidality is a serious condition that covers a range of disorders, from suicidal ideation to suicidal acts. Suicidal thoughts, a primer of the process, can exist undetected, regardless of whether a person is suffering from depression or not.

The World Health Organization (WHO) has reported that globally, the elderly have a greater chance of carrying out successful suicide attempts than any other age group, and the elderly are showing an increasing trend towards higher suicide rates in many countries, including Thailand. ${ }^{1}$ Even though in Thailand, the suicide rate was equal between older and younger adults in 2011, older adults are the fastest growing section of the population in this respect, meaning the number of suicides among this age group is expected to rise considerably in coming years. ${ }^{2}$ As a result, building 
effective strategies and approaches for the prevention of late-life suicide is a major public health priority throughout the world. However, there are a range of factors that impact upon the presence or otherwise of suicidal tendencies, such as whether a person has a psychiatric illness (primarily a depressive disorder), a person's personality and coping style, the presence of a physical illness and pain, and also social stressors and support. ${ }^{3-8}$

It is important to detect suicidality as early as possible when screening as a routine process, and to do this one should administer self-rated questionnaires. Brown reviewed measures used to detect suicidality, both selfrated and clinician-rated. ${ }^{9}$ To do this, the author assessed 31 measures containing from one (Hamilton Rating Scale for Depression, suicide item) to 100 items (suicide opinion questionnaire). Most of these were tested in a psychiatric and medical setting; only eleven measures were examined in a community setting.

In older adults, the geriatric depression scale (GDS) can be used in a wide variety of settings, ranging from outpatient units, to the community and long term care (LTC) facilities. Unfortunately, GDS does not contain any item that directly addresses suicidality. ${ }^{10,11}$

The 9-item Patient Health Questionnaire (PHQ-9) is another measure used to screen for depression in primary care situations, as it measures suicidal ideation. When testing this instrument for suicide, Uebelacker et al found a sensitivity of 0.69 and a specificity of $0.84 .{ }^{12}$

In this study, we used the core symptom index (CSI) in a LTC facility. ${ }^{13}$ The CSI was developed from the Symptom checklist-90 (SCL-90), in order to assess clinical symptoms in adults and the elderly. ${ }^{14,15}$ It has sub-scales related to anxiety, depression, and somatic symptoms. Like the SCL, respondents rate the frequency of their symptoms over the previous week, ranging from "never" to "almost always". This measure aims to explore anxiety, depression, and somatic complaints, which are common in older adults. ${ }^{13}$ More importantly, it contains a suicide item (item two), in which it asks for a response on "suicidal ideation". The aim of this study, therefore, was to determine the test performance of the CSI-item two instrument for the screening of suicide, against the performance of clinician-rated instruments, in particular the Cornell Scale for Depression in Dementia (CSDD) and the suicidality module of the Mini-International Neuropsychiatric Interview (MINIsuicidality). ${ }^{16,17}$ If suitable performance is revealed, the CSI might then be used routinely and also be applied in other geriatric settings.

\section{Materials and methods}

The study was cross-sectional, and was conducted between January and March 2012. The project was approved by the Ethics Committee, Faculty of Medicine, Chiang Mai University and the subjects gave informed consent.

\section{Participants}

The participants were elderly residents (aged 60 and over) at a long-term care facility in Chiang Mai, northern Thailand. All the participants were interviewed by a trained clinical research psychologist and a trained psychiatric nurse, in order to establish a Diagnostic and Statistical Manual of Mental Disorders, Fourth Edition (DSM-IV) diagnosis, using MINI and The Cornell Scale of Depression in Dementia (CSDD). Those who could not finish the interview due to physical disability, psychiatric illness, or cognitive impairment, or could not communicate adequately, were excluded from the study. In total, 80 patients were invited to participate in the study, though three were excluded due to communication problems, four were suffering from severe cognitive impairment and could not provide enough information, and three refused to participate. As a result, 70 were left to participate, and as well as being interviewed, they completed GDS and CSI questionnaires.

\section{Instruments}

\section{CSI}

The CSI is a self-rated instrument designed as a screening and follow-up measurement tool, and was modified from the SCL-90. ${ }^{13-15}$ The instrument consists of 17 items, all of which are based on a 5-point Likert scale and include response values of 0 (never), 1 (rarely), 2 (sometimes), 3 (frequently), and 4 (almost always). The total scores produced can be consistently interpreted; the higher the score, the higher the level of psychopathology present. The CSI consists of three sub-scales: depression, anxiety, and somatic complaints, all of which are common psychiatric problems in elderly patients. The depression sub-scale consists of four items, the anxiety sub-scale consists of six items, and the somatic complaints sub-scale consists of seven items. CSI was previously tested on 41 elderly patients not related to this study, and showed a high level of reliability providing a Cronbach's alpha of 0.87 .

\section{GDS}

The GDS was developed by Yesavage in order to assess the level of depression found among elderly people. ${ }^{18}$ The Thai version of the 15-item GDS (TGDS-15) has demonstrated good psychometric properties when assessing 
geriatric outpatients without cognitive impairment, giving a Cronbach's alpha of $0.82 .{ }^{19}$ This version has 15 items, with each item giving a score of yes or no. The higher the score, the more depression is present. There is no specific question about suicide in the measure, though some items indirectly imply suicidal thoughts. For example, item three asks, "Do you feel that your life is empty?", item seven asks "Do you feel happy most of the time?", item eleven asks "Do you think it is wonderful to be alive?", item 12 asks "Do you feel pretty worthless the way you are now?", and item 14 asks "Do you feel that your situation is hopeless?". ${ }^{20}$ In this study, receiver operating characteristics (ROC) analysis provided an accuracy score of $88 \%$ in terms of the GDS detecting major depressive disorder (MDD), when compared to the MINI, which had an optimal score of $\geq 7$, and yielded a sensitivity of 88 and a specificity of 75 .

\section{CSDD}

The CSDD was developed by Alexopoulos et al, then translated into Thai and tested by Wongpakaran et al. ${ }^{16,21}$ It contains 19 items, covering mood-related signs, behavioral, physical and cyclic functions, and ideational disturbance. Each item can be given a score ranging from 0 (absent) to 2 (severe). The higher the score the greater the level of depression indicated. Item 16, "suicide", includes answers related to "feeling life is not worth living", having had suicidal wishes and having attempted suicide. The questions included under item 16 are as follows: "During the past week, has your relative had any thoughts that life is not worth living or that she/he would be better off dead? And: "Has she/he had any thoughts of hurting or even killing her/himself?" When giving a rating, the interviewer can give a score of 1 for passive suicidal ideation (ie, feels life isn't worth living but has no plans to act), or 2 for active suicidal wishes, and/or any recent suicide attempts, gestures, or plans. However, having a history of suicide attempts, but without showing any signs of current passive or active suicidal ideation, is not scored. In this study, the CSDD scale was used by a trained psychiatric research nurse.

\section{MMSE-Thai 2002}

The MMSE-Thai 2002 has been used to measure cognitive impairment in Thai people, having been modified from the MMSE instrument originally developed by Folstein et al. ${ }^{22}$ The Thai version requires information regarding the participants' education levels in order to create cut-off scores for the cognitive impairment or dementia labels. The total scores range from 0 to 30 and for cognitive impairment or dementia, the cut-off score is 22 . For those who have not completed elementary school level education, the cut-off score is 17 , while for illiterate respondents, the cut-off score is 14 out of a total score of 23 .

\section{MINI suicidality}

The suicidality module of the MINI (version 5) is used to detect suicidality. MINI was originally developed by Sheehan et al. ${ }^{17}$ The Thai version has been validated by Kittiratanapaiboon et al. ${ }^{23}$ In this version, 6-item, dichotomous type responses (yes/no) are applied (in the recent version of Sheehan's Suicidality Tracking Scale [STS], the responses are graded on a 5-rating scale). The item "endorsement" includes the questions: "Over the past month have you thought you would be better off dead or that you wished you were dead?", "Over the past month have you wanted to harm yourself, or to hurt or injure yourself?", “Over the past month have you thought about suicide?", "Over the past month have you planned a suicide attempt?", "Over the past month have you taken active steps to prepare a suicide attempt in which you expected or intended to die?", and "Over the past week, have you injured yourself on purpose?"

\section{Data analysis}

In this study, Pearson's correlation was calculated to establish any association between the CSI-item 2 score, the MINIsuicide module and item 16 of the CSDD. To compare the level of agreement between the MINI-suicide module and item 16 of the CSDD, Gwet's AC1 and Cohen's Kappa were used, using AgreeStat software version 2011.3 (Advanced Analytics, Gaithersburg, MD, USA). ${ }^{24,25}$

The performance of the CSI was examined using ROC analyses of the sensitivity, specificity, positive predictive values (PPV), and negative predictive values (NPV) found. To find the best cut-off score, the Youden index J was used. MedCalc version 12.3 was used for the analysis (MedCalc Software, Mariakerke, Belgium), with univariate analysis used to find predictors for the severity of suicidality. Statistical analysis was conducted using SPSS for Windows version 17 (IBM Corporation, Armonk, NY, USA).

\section{Results}

The mean age and standard deviation (SD) for the residents were 77 and 7.10 respectively. A greater proportion of the participants was female $(61.3 \%)$, and the mean number of years in education was 3.4 years. Only $7 \%$ were living with their spouses. Twenty-four percent of the participants had MDD, while 27\% had suicidal thoughts according to MINI. Thirty-four percent of the participants displayed a risk of 
suicide, though for most of these the risk was minimal $(22.9 \%$ of the $34 \%$ ).

Thirty-seven percent of the sample displayed cognitive impairment. The mean \pm SD figures were $23.97 \pm 4.64$ for the MMSE, 8.51 \pm 2.81 for the GDS, and 11.82 \pm 12.03 for the CSI (Table 1).

In terms of the correlation between item 2 of the CSI and the suicidality items in MINI, as expected there was a high level of correlation between the data gathered from the self-reporting and interview methods with regard to suicidal thoughts, in terms of their degree $(r=0.773, P<0.001)$. Also, a positive correlation was found between other items, these being: "Do you think that you would be better-off dead or wish you were dead?" and "Do you want to harm yourself, or to hurt or injure yourself?" ( $r=0.553, P<0.001$, and $r=0.714$, $P<0.001$, respectively). Likewise, item 2 of the CSI correlated with item 16 of the CSDD $(r=0.626, P<0.001)$.

To examine the level of agreement between measures of suicidal thoughts (those used by different interviewers), Cohen's Kappa and Gwet's AC1 were compared, with a percentage level of agreement calculated. ${ }^{24} \mathrm{~A}$ high level of agreement was found between MINI and CSDD (0.70 for Cohen's Kappa, 0.91 for Gwet's AC1, and a 93\% level of agreement). Using Cohen's Kappa, the level of agreement fell within moderate to good level while when using Gwet's AC1,

Table I Participant socio-demographic and clinical characteristics

\begin{tabular}{|c|c|}
\hline & Value $(n=70)$ \\
\hline \multicolumn{2}{|l|}{ Socio-demographic characteristics } \\
\hline Age: mean \pm SD (minimum-maximum) & $77.01 \pm 7.10(63-94)$ \\
\hline Gender: n (\%) female & $68(61.3)$ \\
\hline $\begin{array}{l}\text { Years of education: mean } \pm \text { SD } \\
\text { (minimum-maximum) }\end{array}$ & $3.37 \pm 3.15(0-12)$ \\
\hline Marital status: $n(\%)$ living with spouse & $5(7)$ \\
\hline \multicolumn{2}{|l|}{ Clinical characteristics } \\
\hline MDD: n (\%) & $17(24.3)$ \\
\hline Suicide ideation by MINI: n (\%) & $8(11.4)$ \\
\hline Suicide by CSDD: $\mathrm{n}(\%)$ & II (I5.7) \\
\hline Suicide score: mean \pm SD (minimum-maximum) & $1.59 \pm 3.34(0-19)$ \\
\hline \multicolumn{2}{|l|}{ Suicide risk: $\mathrm{n}(\%)$} \\
\hline None & $46(65.7)$ \\
\hline Minimal & $16(22.9)$ \\
\hline Moderate & $7(10)$ \\
\hline Severe & I ( I.4) \\
\hline MMSE: mean \pm SD (minimum-maximum) & $23.97 \pm 4.64(12-30)$ \\
\hline Cognitively impaired: n (\%) & $26(37.1)$ \\
\hline GDS: mean \pm SD & $8.5 I \pm 2.8 I$ \\
\hline CSI: mean \pm SD & $11.82 \pm 12.03$ \\
\hline
\end{tabular}

Abbreviations: CSDD, Cornell Scale of Depression in Dementia; CSI, core symptom index; GDS, geriatric depression scale; MDD, major depressive disorder; MINI, Mini-International Neuropsychiatric Interview; MMSE, Mini-Mental Status Examination; SD, standard deviation. it fell in good or almost perfect level, in accordance with the criteria developed by Altman, Landis and Koch. ${ }^{26,27}$

To find predictors of the severity of suicidal thoughts, the CSI, item 2 of the CSI, GDS, and MDD instrument scores were included as independent variables in the univariate analysis. This analysis showed that only item 2 of the CSI predicted the severity of suicidal thoughts $(t=5.849, P<0.001$, $95 \%$ confidence interval $[\mathrm{CI}]=1.382,2.815$, with an effect size [partial Eta Square] of 0.345), while MDD, GDS, and the total CSI score did not (Table 2).

ROC curve analysis was carried out to assess the sensitivity and specificity of the CSI, with a score of at least 1 for item 2 yielding a high sensitivity (100\%) and specificity (90.32\%) for suicidal thoughts in MINI, and with an area under the curve (AUC) of $97 \%$ indicating a high clinical value of the discriminatory power. ${ }^{28} \mathrm{~A}$ score more than 3 for the CSI yielded the highest specificity for both items 2 and 3 of the MINI (Table 3).

\section{Discussion}

To our knowledge, this is the first study that has attempted to detect residents' suicidal thoughts in a LTC facility in Thailand, as reported by both the residents themselves and clinical interviewers. The results here highlight the possibility of screening for suicide in elderly who are LTC facility residents using a brief, easy-to-administer questionnaire. During this study, CSI-item 2 demonstrated an adequate level of validity and so can be recommended as a measure for assessing suicidality when compared to the use of standard interviews.

When compared to previous studies on this topic, this study generated higher levels of sensitivity and specificity than using the PHQ-9 (using item 9 for suicidality) and the GDS-suicide item scale (using four items to imply the presence of suicidal thoughts). ${ }^{20,29-32}$ When using the PHQ-9, Uebelacker et al had a sensitivity of 0.69 and specificity of $0.84 .{ }^{12}$ However, Razykov et al found that item 9 of the PHQ-9 does not accurately screen for the risk of suicide in some settings, though they noted that this may have been due to the timing of the assessment they made, because their study took place over a 12 -month period and the results may not have reflected responses at the time the interviews were held. ${ }^{29}$ Likewise, Walker et al, using the PHQ-9 item 9 to explore this association in cancer patients, found that only one-third of these patients reported suicidal thoughts in subsequent interviews. ${ }^{33}$ However, they were not able to calculate sensitivity and specificity, as those who scored 0 were excluded from the subsequent interviews. The level of 
Table 2 Predictors of the severity of suicidal thoughts (total suicide score)

\begin{tabular}{llllllll}
\hline Parameter & B & $\begin{array}{l}\text { Standard } \\
\text { error }\end{array}$ & t-statistics & & P-value & & \multicolumn{2}{c}{$\mathbf{9 5 \% \text { confidence interval }}$} & $\begin{array}{l}\text { Partial Eta } \\
\text { squared }\end{array}$ \\
\hline Intercept & & & & & Lower bound & Upper bound \\
GDS & 1.487 & 1.453 & 1.024 & 0.310 & -1.414 & 4.388 & 0.016 \\
CSI & 0.076 & 0.122 & 0.626 & 0.534 & -0.167 & 0.319 & 0.006 \\
Item 2 of the CSI & -0.045 & 0.035 & -1.297 & 0.199 & -0.114 & 0.024 & 0.025 \\
Current MDD & 2.099 & 0.359 & 5.849 & 0.000 & 1.382 & 2.815 & 0.345 \\
\hline
\end{tabular}

Abbreviations: B, unstandardized coefficient; CSI, core symptom index; GDS, geriatric depression scale; MDD, major depressive disorder.

agreement found between patients when self-reporting and when interviewed may be influenced by a number of factors, such as the presence of mistaken endorsement (poor validity of the self-reporting instrument), a lack of willingness to report to an interviewer (even if suicidal thoughts do exist), or fluctuating suicidal thoughts. Our results, however, show a high level of agreement between self-reporting mechanisms and interviewer-reporting. This difference between our results and those of other studies may be due to a number of factors, such as the nature of the sample and the level of experience of the interviewer.

In this study, the suicide items of the GDS-15 were found to have a sensitivity of 0.79 , a specificity of 0.80 , and an AUC of 0.83 (standard error 0.031 ), while the CSI was found to have a better test performance, because the items in CSI address suicidal thoughts more than those in the GDS-suicide items. In addition, we found the CSI to be the only predictor of severity effective enough to be used for screening, when compared to the GDS and current MDD instruments. It is interesting to note that in a LTC facility

Table 3 Operating characteristics of the CSI regarding each suicidality item in MINI

\begin{tabular}{|c|c|c|c|c|c|}
\hline & $\begin{array}{l}\text { Area under } \\
\text { the curve (SE) }\end{array}$ & Sensitivity & Specificity & PPV & NPV \\
\hline \multicolumn{6}{|c|}{ MINI suicide item I } \\
\hline$>0$ & $0.770(0.061)$ & $57.89^{a}$ & 94.12 & 78.6 & 85.7 \\
\hline$>1$ & & 36.84 & 96.08 & 77.8 & 80.3 \\
\hline$>2$ & & 26.32 & 100.00 & 100.0 & 78.5 \\
\hline \multicolumn{6}{|c|}{ MINI suicide item 2} \\
\hline$>\mathbf{I}$ & $0.98(0.016)$ & 100.00 & 93.85 & 55.6 & 100.0 \\
\hline$>2$ & & 60.00 & 96.92 & 60.0 & 96.9 \\
\hline$>3$ & & 60.00 & 98.46 & 75.0 & 97.0 \\
\hline \multicolumn{6}{|c|}{ MINI suicide item 3} \\
\hline$>0$ & $0.97(0.016)$ & 100.00 & 90.32 & 57.1 & 100.0 \\
\hline$>1$ & & 75.00 & 95.16 & 66.7 & 96.7 \\
\hline$>2$ & & 50.00 & 98.39 & 80.0 & 93.8 \\
\hline$>3$ & & 50.00 & 100.00 & 100.0 & 93.9 \\
\hline
\end{tabular}

Note: a A number in bold indicates the suggested cut-off score, and its corresponding sensitivity, specificity, PPV, and NPV.

Abbreviations: MINI, the Mini-International Neuropsychiatric Interview; NPV, negative predictive value; PPV, positive predictive value; SE, standard error; CSI, core symptom index. where both depression and cognitive impairment coexist, the results here showed that suicidal ideation was not related to depression, emphasizing the particular characteristics of suicidality found among the residents of LTC home facilities, suggesting that suicidal thoughts may be related to the levels of hopelessness and anxiety being experienced, as well as depression. ${ }^{34}$ This may explain why the CSI was found to be a better predictor than GDS. As we know, elderly people displaying multiple risk factors should be singled-out for special attention; however, suicidal thoughts can come and go and so be unpredictable, meaning it is important for clinicians to be aware of and detect them as soon as possible, in order to develop appropriate interventions. Direct inquiries with regard to suicide, either during health care provider interviews or based on the use of screening questionnaires, should be an important step in any intervention and across all settings.

Further investigations should be carried out to examine the CSI's sensitivity and specificity for MDD, anxiety disorder, and somatoform disorder, in addition to suicide - the condition it was originally developed to measure. As evidence shows from our results, depressed elderly people display more physical symptoms than either their non-depressed counterparts or younger depressed people. ${ }^{15,35}$ In addition to somatic symptoms, anxiety symptoms can also be expressed through somatic complaints or somatization in older men. We believe that the CSI may not only be used with elderly people in the LTC but also in other medical settings. When compared to the outcome measures used by SCL-90, the CSI is easier to use in order to track changes after interventions have taken place, as is the outcome questionnaire- 45 , but is much easier to use than either of these two instruments with elderly respondents. ${ }^{36-38}$

\section{Limitations}

Since the sample size was small and there was only one setting, a larger sample as well as a variety of settings, such as outpatient and inpatient departments should be included in any future investigations. Also, a validity study comparing 
the concurrent validity of this study with other studies, as well as confirmatory factor analysis to explore the instruments' construct validity should both be carried out. In terms of reliability analysis, the CSI needs to be assessed for its stability (test-retest reliability) and also its sensitivity to change, if it is to become an outcome measure that will benefit follow-up interventions, those carried out in addition to screening measures.

\section{Conclusion}

The CSI shows potential for helping to screen for suicidality and in assessing its severity among residents in a long term care facility - those with or without depression or cognitive impairment. It also shows promise in terms of its use as a screening tool for the elderly in other medical settings.

\section{Author contributions}

TW and NW played an equal part in conceiving and designing this study and writing the manuscript. Statistical analysis was performed by TW. Both authors approved the final manuscript.

\section{Acknowledgments}

The authors owe a great debt of gratitude to Ms Kanlaya Kaewsom, our former research nurse, and all the residents and caregivers at TWDCOP for their cooperation during the data collection activities.

\section{Disclosure}

The authors declare that they have no conflicts of interest in this work.

\section{References}

1. WHO. Suicide Prevention (SUPRE). Geneva: World Health Organization; 2013. http://www.who.int/mental_health/prevention/en/. Accessed October 6, 2013.

2. OECD. Society at a Glance Asia/Pacific 2011. OECD Publishing; 2011. Available from: http://www.oecd.org/els/soc/49263450.pdf. Accessed October 6, 2013.

3. Tang WK, Lu JY, Mok V, Ungvari GS, Wong KS. Is fatigue associated with suicidality in stroke? Arch Phys Med Rehabil. 2011;92(8): 1336-1338.

4. Zhang Y, Law CK, Yip PS. Psychological factors associated with the incidence and persistence of suicidal ideation. J Affect Disord. 2011;133(3):584-590.

5. Kar N. Suicidality following a natural disaster. Am J Disaster Med. 2009;5(6):361-368.

6. Fishbain DA, Bruns D, Lewis JE, Disorbio JM, Gao J, Meyer LJ. Predictors of homicide-suicide affirmation in acute and chronic pain patients. Pain Med. 2011;12(1):127-137.

7. Heisel MJ, Links PS, Conn D, van Reekum R, Flett GL. Narcissistic personality and vulnerability to late-life suicidality. Am J Geriatr Psychiatry. 2007;15(9):734-741.
8. Marcinko D, Vuksan-Cusa B. Borderline personality disorder and bipolar disorder comorbidity in suicidal patients: diagnostic and therapeutic challenges. Psychiatr Danub. 2009;21(3):386-390.

9. Brown GK. A Review of Suicide Assessment Measures for Intervention Research with Adults and Older Adults. 2001; Available from: http://www.suicidology.org/c/document_library/get_ file?folderId=235\&name=DLFE-113.pdf. Accessed October 28, 2013.

10. Mitchell AJ, Bird V, Rizzo M, Meader N. Diagnostic validity and added value of the Geriatric Depression Scale for depression in primary care: a meta-analysis of GDS30 and GDS15. J Affect Disord. 2010;125(1-3): 10-17.

11. Mitchell AJ, Bird V, Rizzo M, Meader N. Which version of the geriatric depression scale is most useful in medical settings and nursing homes? Diagnostic validity meta-analysis. Am J Geriatr Psychiatry. 2010;18(12):1066-1077.

12. Uebelacker LA, German NM, Gaudiano BA, Miller IW. Patient health questionnaire depression scale as a suicide screening instrument in depressed primary care patients: a cross-sectional study. Prim Care Companion CNS Disord. 2011;13(1).

13. Wongpakaran N, Wongpakaran T. Prevalence of major depressive disorders and suicide in long-term care facilities: a report from northern Thailand. Psychogeriatrics. 2012;12(1):11-17.

14. Derogatis L. SCL-90-R Administration, Scoring and Procedures Manual-II. Towson: Clinical Psychometric Research; 1983.

15. Wongpakaran T, Wongpakaran N, Boripuntakul T. Symptom checklist-90 (SCL-90) in a Thai sample. J Med Assoc Thai. 2011;94(9): 1141-1149.

16. Alexopoulos GS, Abrams RC, Young RC, Shamoian CA. Cornell Scale for Depression in Dementia. Biol Psychiatry. 1988;23(3): 271-284.

17. Sheehan DV, Lecrubier Y, Sheehan KH, et al. The Mini-International Neuropsychiatric Interview (MINI): the development and validation of a structured diagnostic psychiatric interview for DSM-IV and ICD-10. J Clin Psychiatry. 1998;59 Suppl 20:22-33; quiz 34.

18. Yesavage JA, Brink TL, Rose TL, et al. Development and validation of a geriatric depression screening scale: a preliminary report. J Psychiatr Res. 1982;17(1):37-49.

19. Wongpakaran N, Wongpakaran T, van Reekum R. The use of GDS15 in detecting MDD: a comparison between residents in a Thai longterm care home and geriatric outpatients. J Clin Med Res. 2013;5(2): 101-111.

20. Heisel MJ, Duberstein PR, Lyness JM, Feldman MD. Screening for suicide ideation among older primary care patients. J Am Board Fam Med. 2010;23(2):260-269.

21. Wongpakaran N, Wongpakaran T, van Reekum R. Discrepancies in Cornell Scale for Depression in Dementia (CSDD) items between residents and caregivers, and the CSDD's factor structure. Clin Interv Aging. 2013;8:641-648

22. Folstein MF, Folstein SE, McHugh PR. "Mini-mental state". A practical method for grading the cognitive state of patients for the clinician. J Psychiatr Res. 1975;12(3):189-198.

23. Kittirattanapaiboon $\mathrm{P}, \mathrm{Khamwongpin} \mathrm{M}$. The validity of the mini international neuropsychiatric interview (MINI) - Thai Version 126. J Ment Health Thai. 2005;13(3):126-136.

24. Wongpakaran N, Wongpakaran T, Wedding D, Gwet KL. A comparison of Cohen's Kappa and Gwet's AC1 when calculating inter-rater reliability coefficients: a study conducted with personality disorder samples. BMC Med Res Methodol. 2013;13:61.

25. Gwet KL. Handbook of Inter-Rater Reliability. The Definitive Guide to Measuring the Extent of Agreement among Raters. 2nd edition. Gaithersburg: Advanced Analytics, LLC; 2010.

26. Altman DG. Practical Statistics for Medical Research. London: Chapman and Hall; 1992.

27. Landis J, Koch G. The measurement of observer agreement for categorical data. Biometrics. 1977;33(1):159-174.

28. Faraggi D, Reiser B. Estimation of the area under the ROC curve. Stat Med. 2002;21(20):3093-3106. 
29. Razykov I, Ziegelstein RC, Whooley MA, Thombs BD. The PHQ-9 versus the PHQ-8 - is item 9 useful for assessing suicide risk in coronary artery disease patients? Data from the Heart and Soul Study. J Psychosom Res. 2012;73(3):163-168.

30. Razykov I, Hudson M, Baron M, Thombs BD; Canadian Scleroderma Research Group. Utility of the Patient Health Questionnaire-9 to assess suicide risk in patients with systemic sclerosis. Arthritis Care Res (Hoboken). 2013;65(5):753-758.

31. Donnelly PL, Kim KS. The Patient Health Questionnaire (PHQ-9 K) to screen for depressive disorders among immigrant Korean American elderly. J Cult Divers. 2008;15(1):24-29.

32. O'Riley AA, van Orden KA, He H, Richardson TM, Podgorski C, Conwell. Suicide And death ideation in older adults obtaining aging services. Am J Geriatr Psychiatry. In press.

33. Walker J, Hansen CH, Butcher I, et al. Thoughts of death and suicide reported by cancer patients who endorsed the "suicidal thoughts" item of the PHQ-9 during routine screening for depression. Psychosomatics. 2011;52(5):424-427.
34. Szanto K, Gildengers A, Mulsant BH, Brown G, Alexopoulos GS, Reynolds CF. Identification of suicidal ideation and prevention of suicidal behaviour in the elderly. Drugs Aging. 2002;19(1):11-24.

35. Wongpakaran T, Wongpakaran N, Sirithepthawee U, et al. Interpersonal problems among psychiatric outpatients and non-clinical samples. Singapore Med J. 2012;53(7):481-487.

36. Wongpakaran T, Wongpakaran N, Boripuntakul T. Symptom checklist-90 (SCL-90) in a Thai sample. J Med Assoc Thai. 2011;94(9) 1141-1149.

37. Hardy AO, Tracey TJ, Glidden-Tracey C, Hess TR, Rohlfing JE. Interpersonal contribution to outcome: The relation of interpersonal distress and symptomatic improvement as a result of psychotherapy. Clin Psychol Psychother. 2011;18(3):225-233.

38. Lo Coco G, Chiappelli M, Bensi L, Gullo S, Prestano C, Lambert MJ The factorial structure of the Outcome Questionnaire-45: a study with an Italian sample. Clin Psychol Psychother. 2008;15(6):418-423.
Clinical Interventions in Aging

\section{Publish your work in this journal}

Clinical Interventions in Aging is an international, peer-reviewed journal focusing on evidence-based reports on the value or lack thereof of treatments intended to prevent or delay the onset of maladaptive correlates of aging in human beings. This journal is indexed on PubMed Central, MedLine, the American Chemical Society's 'Chemical Abstracts

\section{Dovepress}

Service' (CAS), Scopus and the Elsevier Bibliographic databases. The manuscript management system is completely online and includes a very quick and fair peer-review system, which is all easy to use. Visit http://www.dovepress.com/testimonials.php to read real quotes from published authors. 